Aim of the study: To investigate the feasibility of enhanced recovery after surgery (ERAS) protocol for patients with primary peritoneal carcinomatosis (PC) undergoing cytoreductive surgery with hyperthermic intraperitoneal chemoperfusion (HIPEC) based on the length of hospital stay (LOS), return of bowel function, the incidence of postoperative complications, and quality of life (QLQ) analysis.

Material and methods: The study included a total of 37 patients with primary PC of different origin, who underwent cytoreductive surgery plus HIPEC. Patients were divided into 2 groups: Group I (nonERAS) - 20 patients and Group II (ERAS) - 17 patients.

Results: The median LOS in Group (nonERAS) $(12.35 \pm 3.9)$ was longer than in Group II (ERAS) $(6.8 \pm 1.9)$ $(p<0.01)$. The use of the ERAS protocol significantly contributed to the faster return of bowel function (peristalsis and stool) in the postoperative period $(p<0.01)$. There was no statistically significant difference in the incidence of postoperative complications between the ERAS and nonERAS groups, which supports its clinical safety. Improved QLQ according to the obtained data has also been achieved due to the introduction of the principles of the ERAS protocol.

Conclusions: The obtained results prove the expediency and feasibility of the implementation of the ERAS protocol among patients undergoing cytoreductive surgery in combination with HIPEC.

Key words: peritoneal carcinomatosis, HIPEC, cytoreductive surgery, ERAS.

Contemp Oncol (Pozn) 2021; 25 (2): 133-139 DOI: https://doi.org/10.5114/wo.2021.107441

\section{Implementation of the enhanced recovery after surgery protocol for patients with peritoneal carcinomatosis undergoing cytoreductive surgery and hyperthermic intraperitoneal chemoperfusion}

Oleksandr Ivanovych Tkachenko, Sergii Hennadiiovych Chetverikov, Oleksandr Vadymovych Bondar, Viacheslav Yevheniiovych Maksymovskyi, Mykhailo Chetverikov, Valeriia Volodymyrivna Chetverikova-Ovchynnyk

Odessa National Medical University, Odessa, Ukraine

\section{Introduction}

Primary peritoneal carcinomatosis (PC) is a metastatic lesion of the peritoneum with a malignant tumour of different origin. Primary PC remains an urgent problem for timely diagnosis and treatment worldwide.

The term 'peritoneal carcinomatosis' was first coined in 1931 by Sampson of Albany Medical College to describe the implantation metastasis of ovarian cancer to the peritoneal cavity [1].

Primary PC was regarded as a hopeless and terminal stage of oncological decease with a negative prognosis for many decades. Surgical treatment was not considered. Palliative systemic chemotherapy has remained the only acceptable specific treatment. The median survival of patients with PC treated with the best supportive care usually does not exceed 3-14 months, depending on the origin of the primary tumour [2-5].

The primary site of cancerous implants dissemination in the peritoneum mostly are malignant gynaecological and gastrointestinal tumours. A similar mechanism of lesion is seen in malignant peritoneal mesothelioma. It is acknowledged that the incidence of synchronous PC in patients with colorectal cancer, according to various data, is $5-15 \%$ [2-4], for gastric cancer about $14-20 \%[2,6]$, and for ovarian cancer $70-80 \%[2,7]$.

The pattern of PC genesis is explained by the biological properties of primary tumours and features of the histological structure of the peritoneum, which allows us to consider the process as locoregional (the theory of "seed" and "soil" by Peget) [8]. Implantation the theory of PC provides the possibility of effective surgical treatment, which increases the quality of life (QLQ) and overall survival of patients.

A paradigm shift in the treatment of PC patients was proposed in 1985 by P.H. Sugarbaker. He described a radical combined approach in the treatment of PC, the goal of which is to remove the maximum possible amount of macroscopically visible tumour mass. In 1994, Elias D. introduced the concept of hyperthermic intraperitoneal chemoperfusion (HIPEC), which affects the microscopic residual tumour mass in patients with PC [3].

The combination of optimal cytoreductive surgery and HIPEC is currently proven as pathogenetically reasonable for the treatment of PC of different primary origin, especially when there are no haematogenous metastases. 
Perioperative practice of PC management in cytoreductive surgery (CRS) and HIPEC differs among different oncology centres due to the lack of a unified approach, the small amount of patients, different volumes of surgery performed, and the lack of standardization of chemoperfusion procedures.

The enhanced recovery after surgery (ERAS) protocol aims to standardize and optimize perioperative care for patients with PC. It includes a holistic and systematic approach to improve surgical outcomes [9-11].

\section{Aim of the study}

To investigate the feasibility of the ERAS protocol for patients with primary PC undergoing cytoreductive surgery with HIPEC based on the length of hospital stay (LOS), return of bowel function, the incidence of postoperative complications, and QLQ analysis.

\section{Material and methods}

The study included a total of 37 patients (8 male, $29 \mathrm{fe}$ male) with primary PC of different origin, who underwent cytoreductive surgery plus HIPEC, dynamic monitoring and control at the clinical base of the Surgery Department nr 3 of Odessa National Medical University during the period 2016-2021. Patients were divided into 2 groups:

- Group I (nonERAS) - 20 patients, who were treated before the introduction of the principles of the ERAS protocol (2016-2018);

- Group II (ERAS) - 17 patients, who were treated in accordance with the perioperative use of the ERAS protocol (2018-2021) (Table 1).

The ERAS protocol included a perioperative approach to the treatment of patients by the multidisciplinary team including oncological surgeons, anaesthetists, clinical oncol-

Table 1. Characteristics of patients in Group I (non-enhanced recovery after surgery) and Group II (enhanced recovery after surgery)

\begin{tabular}{lcc} 
Characteristics & $\begin{array}{c}\text { Group I } \\
\text { (nonERAS), } \\
n=20\end{array}$ & $\begin{array}{c}\text { Group II } \\
\text { (ERAS), } \\
n=17\end{array}$ \\
\hline PCI score + A3: A14 & $11.7 \pm 4.07$ & $10.9 \pm 3.7$ \\
Achieved CC-0 or CC-1 & $18(90 \%)$ & $15(88 \%)$ \\
Laparotomy & $17(85 \%)$ & $11(65 \%)$ \\
Laparoscopy & $3(15 \%)$ & $6(35 \%)$ \\
Total or subtotal peritonectomy & $9(45 \%)$ & $7(41 \%)$ \\
Intestinal anastomoses & $13(65 \%)$ & $12(71 \%)$ \\
Ostomy & $5(25 \%)$ & $4(23 \%)$ \\
Splenectomy & $3(15 \%)$ & $3(18 \%)$ \\
Liver resection & $4(20 \%)$ & $5(29 \%)$ \\
Diaphragm repair & $3(15 \%)$ & $4(23 \%)$ \\
Intraoperative blood loss, ml & $354 \pm 132$ & $326 \pm 104$ \\
Operative time, $h$ & $6.3(3.8-8.5)$ & $6.5(4.2-9.1)$ \\
Abdominal drains & $4.3(3-6)$ & $1.4 \pm(0-3)$ \\
Central vein & $15(75 \%)$ & $5(29 \%)$ \\
Nasogastric/duodenal tube & $12(60 \%)$ & $2(12 \%)$ \\
Epidural placement & $5(25 \%)$ & $14(82 \%)$ \\
\hline
\end{tabular}

ogists, and physicians and urological, gynaecological, and vascular surgeons if necessary. The perioperative approach included preoperative, intraoperative and postoperative management. Preoperative care was individualised and depended on patients' age, comorbid diseases, functional status, tumour burden, albumin rate, and the presence of preoperative anaemia.

Pre-operative management included preadmission counselling, smoking and alcohol cessation, anaemia and hypoalbuminaemia screening, prehabilitation, nutritional supplementation, bowel preparation, pre-anaesthetic medication, and short preoperative fasting. Patients, preparing for CRS + HIPEC received mixed-type educational information about the operation and the recovery period on the preadmission counselling to decrease anxiety and improve psychological outcomes. On the first visit the patients were offered alcohol cessation, and smokers were recommended to start nicotine replacement therapy at least 3 weeks before the surgery. Patients were screened for anaemia and hypoalbuminaemia as early as was possible before the surgery and received appropriate correction if necessary. A prehabilitation programme of physical exercise was indicated routinely in Group II. In the preoperative period during the 2 weeks before the planned surgery patients began a high-protein diet, as well as a carbohydrate diet for 3 days before surgery. Pre-anaesthetic medication included multimodal pain medications and anxiolytics. Intestinal decontamination was performed according to standard methods, and there was no mechanical preparation of the bowel.

Intraoperative management included antimicrobial prophylaxis, skin preparation, protective mechanical ventilation, cardiac output monitoring, use of neuromuscular antagonists, a multimodal anaesthesia including epidural analgesia, prevention of hypo- and hyperthermia, rational infusion therapy (concept of goal-directed fluid therapy), early extubation, no routine central vein catheterization, minimum drain number in the abdominal cavity, and no routine thoracic draining. The temperature was monitored with an oesophageal temperature probe. To prevent intraoperative hypothermia warm intravenous and irrigation fluids were used. To prevent intraoperative hyperthermia during the HIPEC procedure cool packs were used. Epidural analgesia for 3 days or more was used routinely to reduce the need for opioids and to hasten the return of bowel function.

Postoperative management included non-prophylactic nasogastric drainage (only in the case of gastric resection), early removal of the urinary catheter, adequate multimodal postoperative anaesthesia, including T-block and epidural analgesia, nutrition care, monitoring of blood glucose, prophylaxis against thromboembolism (mechanical and pharmacological), early mobilisation and verticalisation of patients, and early drain removal from the abdominal cavity. The urinary catheter was removed on the second postoperative day if there was no bladder resection. Early liquid enteral nutrition of patients with therapeutic protein-carbohydrate mixtures was used. Mobilisation of the patients started on the surgery day and verticalization on the next day to prevent thrombosis and postoperative pneumonia and to hasten the return of bowel function. 
The stage of the tumour process was determined according to the International Classifications TNM $7^{\text {th }}$ and $8^{\text {th }}$ edition (according to the date of the initial diagnosis). Clinical, laboratory, and instrumental examination of patients was performed according to the recommendations of the National Comprehensive Cancer Network, European Society for Medical Oncology, European Society of Gynaecological Oncology, and European Society of Surgical Oncology. The diagnosis of all patients was confirmed histologically and immunohistochemically. Phase contrast imaging (PCI) was determined by preoperative intrascopic methods (computed tomography with intravenous contrast and/or magnetic resonance imaging using diffuse diffusion) and intraoperatively for all patients [12].

The extent of cytoreductive surgery was determined depending on the spread of the tumour mass. Intraoperative characteristics in both groups (completeness of cytoreduction, operative time, intraoperative blood loss), LOS, the incidence of early (up to 7 days after surgery) and late (8-30 days after surgery) postoperative complications grade II-IV by Clavien-Dindo, postoperative mortality, and return of bowel function (peristalsis and stool) after surgery were estimated (Table 2,3 ). The analysis of the QLQ of patients was performed using the European Organization for Research and Treatment of Cancer (EORTC) QLQ Core 30 (C30) scale version 3 before the surgery, on the third day after surgery, and every 10 days for 1 month (Table 4).

The HIPEC procedure was performed in patients of both groups using the RanD Performer HT system by closed abdomen method. During the procedure, the flow rate, the volume of perfusion, and 6-channel control of the perfusion solution pressure in the system were monitored. The procedure of the HIPEC was standard for all patients. Abdominal perfusion (perfusion volume depend on the patient weight, $60 \mathrm{~mL} / \mathrm{kg}$ ) with a solution containing cisplatin $\left(50 \mathrm{mg} / \mathrm{m}^{2}\right)$ and doxorubicin $\left(15 \mathrm{mg} / \mathrm{m}^{2}\right)$ was performed at $41^{\circ} \mathrm{C}$ for 90 minutes. Sodium thiosulphate was administered at the beginning of perfusion as an intravenous bolus $\left(7.5 \mathrm{~g} / \mathrm{m}^{2}\right)$ followed by continuous infusion $\left(25 \mathrm{~g} / \mathrm{m}^{2}\right)$ for 12 hours to reduce the nephrotoxic effect of chemotherapeutic drugs.

The condition of patients in both groups was assessed by analysing clinical, instrumental, and laboratory parameters. After CRS + HIPEC on days 2 and 5 and every 10 days for 1 month after surgery, electrocardiogram and blood tests were performed.

Comparison of patients by the criteria represented by numerical variables was performed using the $U$ Mann-Whitney test. Pearson's consistency criterion $\chi^{2}$ was used in the groups to compare frequencies. Fisher's exact 2-sided criterion was used to determine the relationship.

\section{Results}

The mean age of patients was $55.4 \pm 8.6$ years (Group I: $56.3 \pm 8.7$ years, Group II: $54.5 \pm 8.4$ years $-p>0.05$ ).

Patients in both groups were analysed for alimentary status, family history, anaemia, and comorbidities (mixed comorbidities, cardiovascular diseases, respiratory diseases, urinary pathology, neuroendocrine pathology). Statisti-
Table 2. Early postoperative complications in Group I (non-enhanced recovery after surgery) and Group II (enhanced recovery after surgery) grade II-IV according to Clavien-Dindo

\begin{tabular}{|lcc|}
\hline $\begin{array}{l}\text { Early postoperative complications } \\
\text { (1-7 day), } n \text { (\%) }\end{array}$ & $\begin{array}{c}\text { Group I } \\
\text { (nonERAS), } \\
n=20\end{array}$ & $\begin{array}{c}\text { Group II } \\
\text { (ERAS), } \\
n=17\end{array}$ \\
\hline Bowel perforation (acute ulcer) & $0(0 \%)$ & $1(5.8 \%)$ \\
\hline Coagulopathic bleeding & $1(5 \%)$ & $0(0 \%)$ \\
\hline Anastomotic leak & $3(15 \%)$ & $2(11.8 \%)$ \\
\hline Relaparotomy & $3(15 \%)$ & $2(11.8 \%)$ \\
\hline Eventration & $1(5 \%)$ & $1(5.8 \%)$ \\
\hline Ileus & $5(25 \%)$ & $1(5.8 \%)$ \\
\hline Infectious complications & $3(15 \%)$ & $2(11.8 \%)$ \\
\hline Hyperthermia & $2(10 \%)$ & $3(17.6 \%)$ \\
\hline Acute renal failure & $2(10 \%)$ & $1(5.8 \%)$ \\
\hline Acute liver failure & $1(5 \%)$ & $1(5.8 \%)$ \\
\hline Pleural effusion & $3(15 \%)$ & $1(5.8 \%)$ \\
\hline Pneumothorax & $1(5 \%)$ & $0(0 \%)$ \\
\hline Pulmonary artery thromboembolism & $2(10 \%)$ & $0(0 \%)$ \\
\hline Postoperative pneumonia & $2(10 \%)$ & $0(0 \%)$ \\
\hline Deep vein thrombosis & $0(0 \%)$ & $1(5.8 \%)$ \\
\hline Cerebrovascular disorders & $1(5 \%)$ & $0(0 \%)$ \\
\hline Anemia & $4(20 \%)$ & $3(17.6 \%)$ \\
\hline Perforation of gastric ulcer & $1(5 \%)$ & $0(0 \%)$ \\
\hline
\end{tabular}

Table 3. Late postoperative complications in Group I (non-enhanced recovery after surgery) and Group II (enhanced recovery after surgery) grade II-IV according to Clavien-Dindo

\begin{tabular}{|lcc|}
$\begin{array}{l}\text { Late postoperative } \\
\text { complications (8-30 day), } \\
n \text { (\%) }\end{array}$ & $\begin{array}{c}\text { Group I } \\
\text { (nonERAS), } \\
n=20\end{array}$ & $\begin{array}{c}\text { Group II } \\
\text { (ERAS), } \\
n=17\end{array}$ \\
\hline $\begin{array}{l}\text { Anastomotic leak } \\
\text { Intestinal fistula }\end{array}$ & $1(5 \%)$ & $0(0 \%)$ \\
\hline Infectious complications & $2(10 \%)$ & $0(0 \%)$ \\
\hline Eventration & $1(5 \%)$ & $0(0 \%)$ \\
\hline Ventral hernia & $2(10 \%)$ & $1(5.8 \%)$ \\
\hline Anemia & $3(15 \%)$ & $1(5.8 \%)$ \\
\hline Gastric ulcer bleeding & $1(5 \%)$ & $0(0 \%)$ \\
\hline Deep vein thrombosis & $1(5 \%)$ & $0(0 \%)$ \\
\hline Hospital-acquired pneumonia & $2(10 \%)$ & $0(0 \%)$ \\
Acute myocardial infarction & $0(0 \%)$ & $1(5.8 \%)$ \\
\hline Acute renal failure & $1(5 \%)$ & $1(5.8 \%)$ \\
Acute liver failure & $1(5 \%)$ & $0(0 \%)$ \\
\hline Death & $1(5 \%)$ & $0(0 \%)$
\end{tabular}

cal homogeneity of groups $(p>0.05)$ was determined for each of the indicators. Among both groups, there was no statistically significant difference in the mean $\mathrm{PCl}$, intraoperative characteristics of patients, or the extent of surgery $(p>0.05)$ (Table 1)

Patients with primary PC included in the study were diagnosed as follows: 


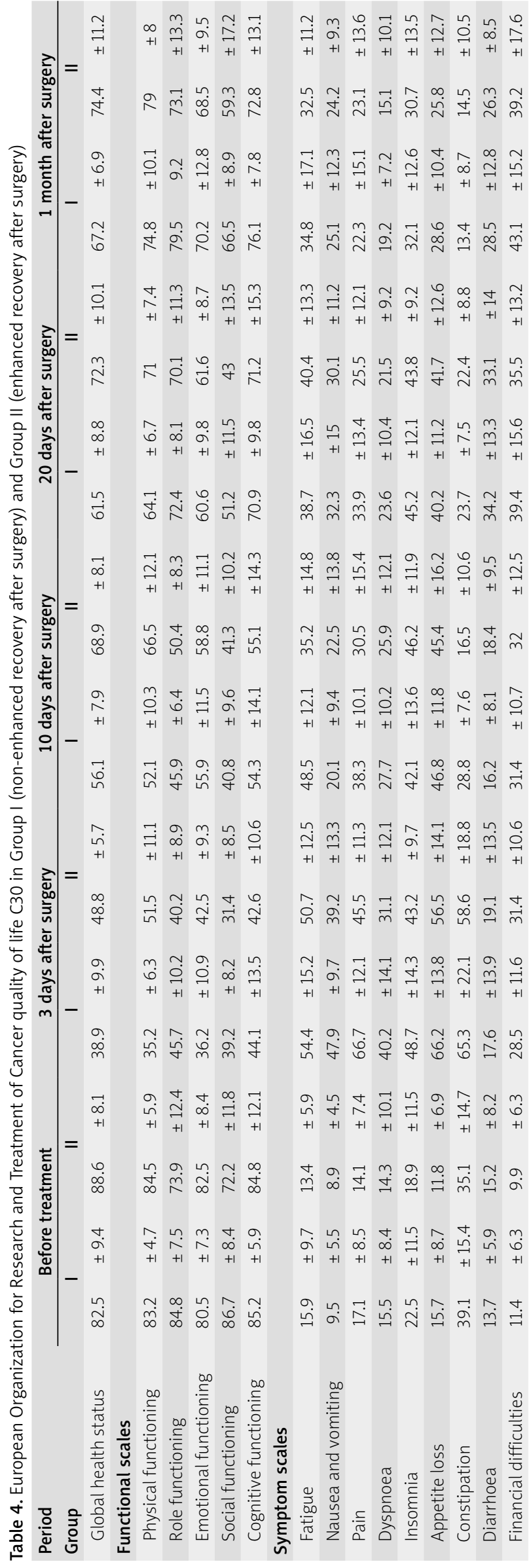

- 15 epithelial ovarian carcinoma (Group I - 8 patients, Group II - 7),

- 9 colorectal adenocarcinoma (Group I - 5 patients, Group II-4),

- 6 gastric adenocarcinoma (Group I - 4 patients, Group II-2),

- 7 malignant peritoneal mesothelioma (Group I - 3 patients, Group II -4).

The median LOS in Group I (nonERAS) was $12.35 \pm 3.9$ (5-32) days, which is longer than in Group II (ERAS) $6.8 \pm$ 1.9 (4-17). Comparing both groups according to the criterion Mann-Whitney, Ukr. = $105>$ Uemp. $=41$, the difference is statistically significant $(p<0.01)$.

When estimating the median duration of recovery of digestive function, the peristalsis recovered after 3.6 (1-6) days in Group I versus 1.7 (1-4) days in Group II (Ukr. = 105 $>$ Uemp. = 39). Defecation in Group I was observed after 5.6 (3-10) days versus 3.4 (2-8) days in Group II (Ukr. = 105 $>$ Uemp. =55). The difference of the return of the bowel function (peristalsis and stool) in the compared groups is statistically significant $(p<0.01)$. The use of the ERAS protocol significantly contributed to the faster restoration of intestinal function in the postoperative period.

Postoperative complications grade II-IV according to Clavien-Dindo classification occurred among 35\% of patients ( 7 patients) (3 patients in the early postoperative period, 4 in the late postoperative period) in Group I (nonERAS) and among $23.5 \%$ of patients (4 patients) ( 3 patients in the early postoperative period, 1 in the late postoperative period) in Group II (ERAS). There was no significant difference according to Fisher's exact bilateral test $(p>0.05)$, i.e. the ERAS protocol did not increase the incidence of postoperative complications among the analysed patients. The list and incidence of early and late postoperative complications in Group I (nonERAS) and Group II (ERAS) are given in Table 2 and 3.

Stoma was formed in 25\% (5) patients in Group I and 23\% (4) in Group II.

There were no intraoperative and early postoperative deaths in both groups. In the late postoperative period in Group I (nonERAS) 1 patient died due to the development of acute hepato-renal failure.

EORTC QLQ-C30 questionnaire (data presented on Fig. 1) were analysed using the $U$ Mann-Whitney test in the preoperative period. There was no significant difference in the global health status of patients, all symptom scales, and scales of physical, emotional, and cognitive functioning. (Ukr. < Uemp. at a significance level of $p=0.05$ ) before the surgery.

Indicators of role and social functioning in Group II (ERAS) were significantly lower than in Group I (nonERAS) at each time point of the survey $(p<0.05)$ (Fig. 1).

On the third postoperative day the indicators of global health status and physical functioning in Group I (nonERAS) were lower than in Group II (ERAS) $(p<0.05)$ (Fig. 2). Symptomatic scales of pain, nausea and vomiting, loss of appetite, and constipation in Group II (ERAS) showed significantly better results than in Group I (nonERAS) (Fig. 2, 3). Similar results were obtained on the $10^{\text {th }}$ and $20^{\text {th }}$ postoperative day (Fig. 2, 3). Differences in the data 
of the symptom scales 1 month after surgery between the 2 groups were statistically insignificant (Ukr. < Uemp. at a significance level of $p=0.05$ ), but the global health status and physical functioning remained higher in Group II (ERAS) (Fig. 1)

\section{Discussion}

The study demonstrated the clinical safety of the ERAS protocol in patients who underwent cytoreductive surgery and HIPEC, compared to the standard perioperative approach (Fig. 4).

The safety of new methods of treatment and their impact on the QLQ of patients precedes the assessment of cancer results. The study included patients with oncological processes of different primary localization, who underwent cytoreductive surgery of the maximum possible extent and HIPEC according to the standardized method. Aggressive surgery approach, toxic effects of chemotherapy, and side effects of hyperthermia lead to high rates of postoperative complications and mortality among patients. ERAS protocols are a combination of procedure-specific evidence-based perioperative strategies that work synergistically to improve the recovery after surgery. This result can be achieved only with a multimodal and multidisciplinary approach to the treatment of each patient. The change to the 'usual' model of perioperative care among the patients led to a decrease in the median LOS and the rapid return of bowel function. The positive effect of ERAS, i.e. return of the bowel function, was evaluated because it is important to reduce the incidence of postoperative complications (ileus, anastomosis leak, intestinal fistulas, etc.) and improve the quality of life. The increase in QLQ in patients treated with ERAS was maintained from the third postoperative day and continued for 1 month after surgery. Significantly lower values of role and social functioning scales in Group II (ERAS) compared to Group I (nonERAS) in the preoperative and subsequent stages of treatment may be due to the epidemiological situation, which imposed forced restrictions on the behaviour of patients in Group II during the period 2020-2021. Based on the obtained data, the introduction of the ERAS protocol reduced the incidence of early and late postoperative complications among the analysed patients ( $23.5 \%$ vs. $35 \%$ ), but the difference is insignificant. Thus, the clinical safety of ERAS implementation for patients after CRS and HIPEC has been proven.

Similar results were obtained in the study of Lu et al. The authors analysed the LOS, postoperative complications and the occurrence of renal dysfunction among patients after CRS and HIPEC with mitomycin C using the ERAS protocol [13]. The median LOS was 6 days in the ERAS group and 9 days in the nonERAS group, which is lower than the data obtained in this study. These results can be explained by the difference in the HIPEC procedure and chemotherapy (low toxicity of mitomycin C comparing to the combination of platinum-therapy and doxorubicin).

Siddharthan et al. did not show a significant difference in the incidence of postoperative complications of grade

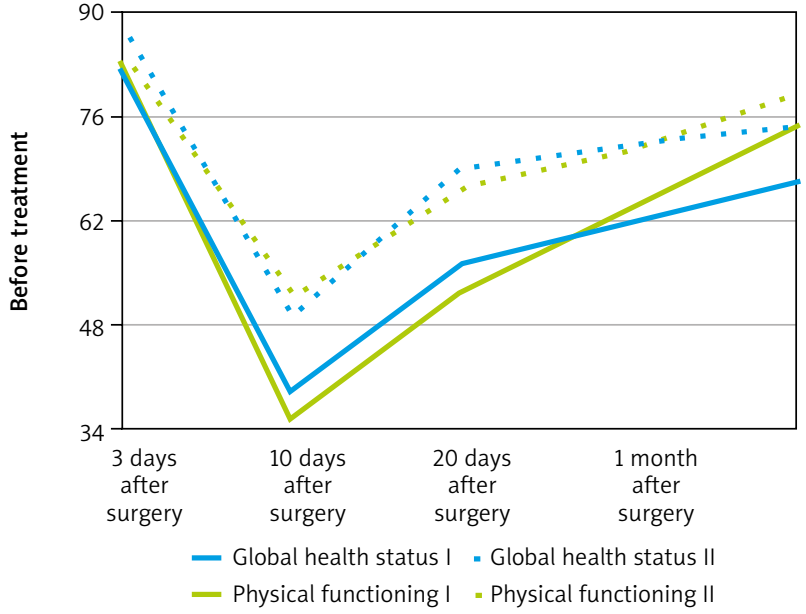

Fig. 1. European Organization for Research and Treatment of Cancer quality of life C30 - perioperative graph of global health status and physical functioning in Group I (non-enhanced recovery after surgery) (solid lines) and Group II (enhanced recovery after surgery) (dotted lines)

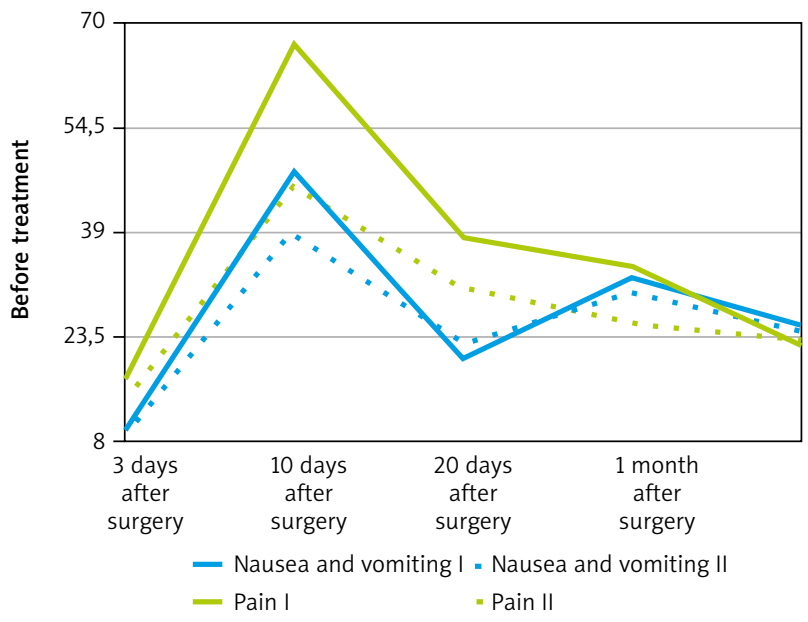

Fig. 2. European Organization for Research and Treatment of Cancer quality of life C30 - perioperative graph of nausea/vomiting and pain in Group I (non-enhanced recovery after surgery) (solid lines) and Group II (enhanced recovery after surgery) (dotted lines)

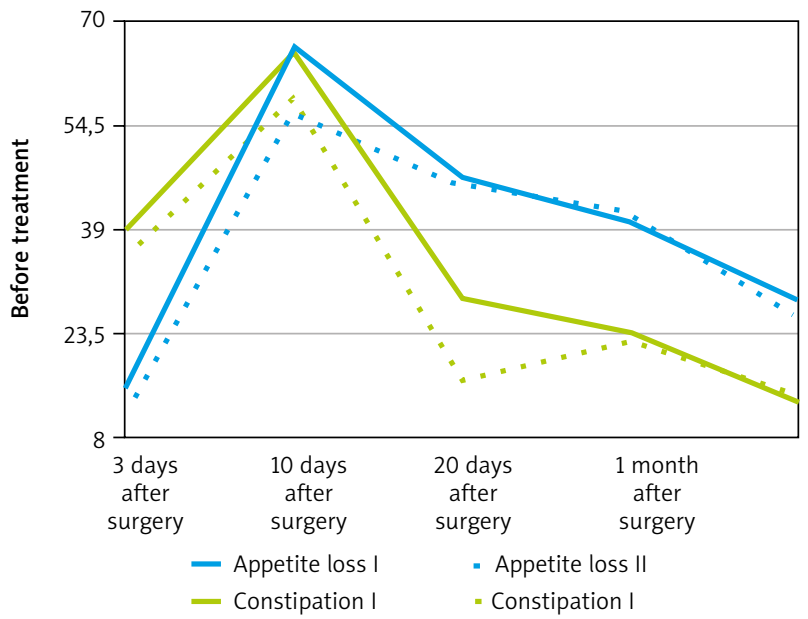

Fig. 3. European Organization for Research and Treatment of Cancer quality of life C30 - perioperative graph of appetite loss and constipation in Group I (non-enhanced recovery after surgery) (solid lines) and Group II (enhanced recovery after surgery) (dotted lines) 


\section{(1) ERAS}

for patients with peritoneal carcinomatosis undergoing cytoreductive surgery + HIPEC

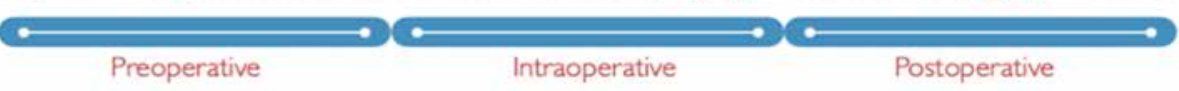

Length of hospital stay

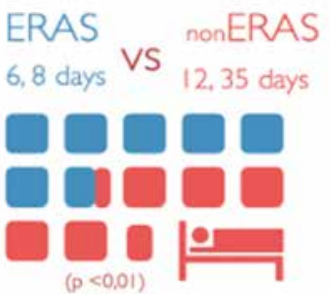

Return of bowel function
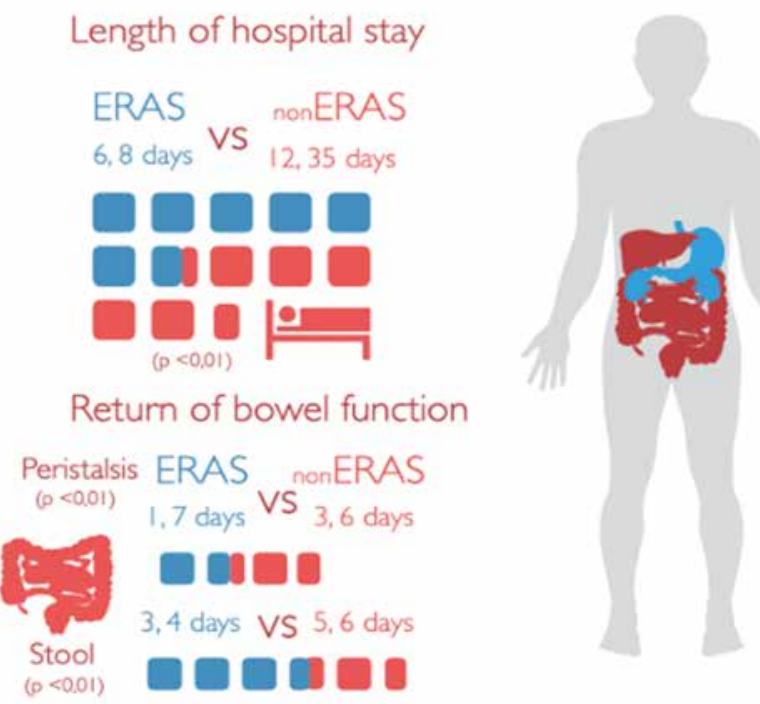

Postoperative complications
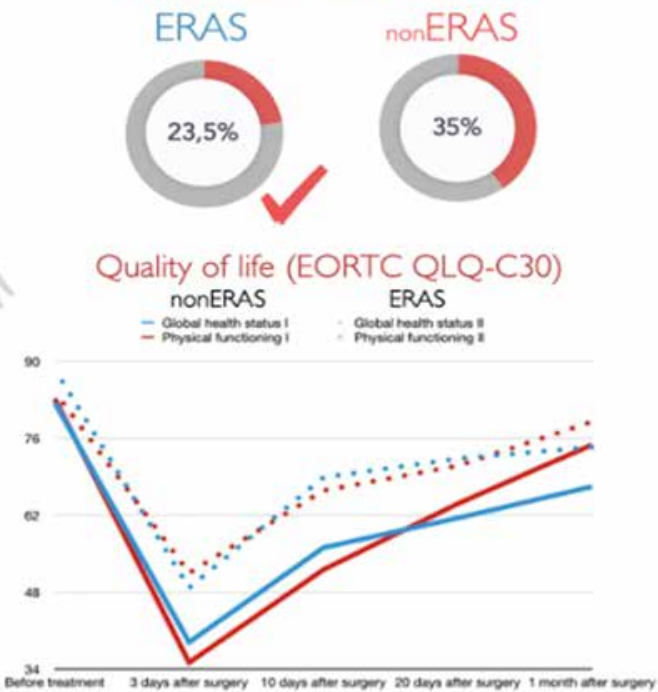

Table 4. European Organization for Research and Treatment of Cancer quality of life C30 in Group I (non-enhanced recovery after surgery) and Group II (enhanced recovery after surgery)

III-IV among patients treated with and without the ERAS protocol after cytoreductive surgery + HIPEC with mitomycin C [14].

Webb et al. in their investigation also showed a decrease in the duration of the hospital stay and the incidence of postoperative complications after the introduction of ERAS principles [15].

In contrast, in the research of Eng et al. 2372 cytoreductive surgeries were performed in many institutions for primary and recurrent PC of various aetiologies, which in most cases were accompanied by HIPEC. Fluctuations in the frequency of complications among patients were explained by the characteristics of patients and the disease rather than the treatment approach in a particular institution [16].

A limitation of this study is the small number of patients, because the standard and unified approach was organized in a single institution.

A positive aspect of the study is the use of a specific cancer questionnaire, which due to the multimodal assessment of QLQ allowed us to objectify changes in the condition and well-being of patients, which allows a more comprehensive assessment of treatment.

\section{Conclusions}

The obtained results prove the expediency and feasibility of the implementation of the ERAS protocol among patients undergoing CRS in combination with HIPEC.

The shorter LOS due to the ERAS protocol allows patients to start rehabilitation and subsequent stages of special treatment earlier, to reduce the economic costs.
Improved QLQ and comfort of cancer patients has also been achieved due to the introduction of the principles of the protocol.

There was no statistically significant difference in the incidence of postoperative complications between ERAS and nonERAS groups, which supports its clinical safety.

Successful implementation of the ERAS protocol requires a coordinated approach of a multidisciplinary team, which begins with the preoperative visit and planning of the surgery.

The authors declare no conflict of interest.

\section{References}

1. Desai JP, Moustarah F. Peritoneal metastasis. [Updated 2021 Jan 10]. In: StatPearls [Internet]. Treasure Island (FL): StatPearls Publishing 2021. Available from: https://www.ncbi.nlm.nih.gov/ books/NBK541114/.

2. Zakharenko AA, Zaitcev DA, Belyaev MA, et al. Modern strategy in treatment of peritoneal carcinomatosis. Sci Notes Pavlov Univ 2017; 24: 7-12

3. Sushkov OI, Achkasov SI. Peritoneal colorectal carcinomatosis. Approaches to treatment (review). Coloproctol 2016; 4: 69-79.

4. Dong XD. Right sided colon cancer and peritoneal carcinomatosis. Ann Laparoscopic Endoscopic Surg 2019; 4: 72.

5. Yarema R, Ohorchak M, Hyrya P, et al. Gastric cancer with peritoneal metastases: efficiency of standard treatment methods. World J Gastrointest Oncol 2020; 12: 569-581.

6. Wang Z, Chen J, Liu J, et al. Issues on peritoneal metastasis of gastric cancer: an update. World J Surg Oncol 2019; 17: 215.

7. Van Baal JOAM, van Noorden CJF, Nieuwland R, et al. Development of peritoneal carcinomatosis in epithelial ovarian cancer: a review. J Histochem Cytochem 2018; 66: 67-83. 
8. Akhtar M, Haider A, Rashid S, Al-Nabet ADMH. Paget's "Seed and Soil" theory of cancer metastasis. Adv Anat Pathol 2019; 26: 69-74.

9. Hübner M, Kusamura S, Villeneuve L, et al. Guidelines for perioperative care in cytoreductive surgery (CRS) with or without hyperthermic IntraPEritoneal chemotherapy (HIPEC): enhanced recovery after surgery (ERAS ${ }^{\circledR}$ ) Society Recommendations - Part I: preoperative and intraoperative management. Eur I Surg Oncol 2020; 46: 2292-2310

10. Hübner M, Kusamura S, Villeneuve L, et al. Guidelines for perioperative care in cytoreductive surgery (CRS) with or without hyperthermic IntraPEritoneal chemotherapy (HIPEC): enhanced recovery after surgery (ERAS $\left.{ }^{\circledR}\right)$ Society Recommendations - Part II: postoperative management and special considerations. Eur J Surg Oncol 2020; 46: 2311-2323.

11. Solanki SL, Mukherjee S, Agarwal V, et al. Society of onco-anaesthesia and perioperative care consensus guidelines for perioperative management of patients for cytoreductive surgery and hyperthermic intraperitoneal chemotherapy (CRS-HIPEC). Indian J Anaesth 2019; 63: 972-987.

12. Chetverikov S, Zavoloka S, Onyshchenko V, Chetverikov M, Chetverikova-Ovchynnyk V. Magnetic resonance imaging with diffuse weighted imaging and computed tomography with intravenous contrast in staging of disseminated ovarian, stomach, colorectal cancer. EUREKA Health Sci 2019; 6: 59-65. Available from: http:// dx.doi.org/10.21303/2504-5679.2019.001071.

13. Lu PW, Fields AC, Shabat G, et al. Cytoreductive surgery and HIPEC in an enhanced recovery after surgery program: a feasibility study. J Surg Res 2020; 247: 59-65.

14. Siddharthan R, Dewey E, Billingsley K, Gilbert E, Tsikitis VL. Feasibility and benefits of an enhanced recovery after surgery protocol for patients undergoing cytoreductive surgery and heated intraperitoneal chemotharpy: a single institution experience. Am J Surg 2020; 219: 1073-1075.

15. Webb C, Day R, Velazco CS, et al. Implementation of an enhanced recovery after surgery (ERAS) program is associated with improved outcomes in patients undergoing cytoreductive surgery and hyperthermic intraperitoneal chemotherapy. Ann Surg Oncol 2020; 27: 303-312.

16. Eng OS, Blakely AM, Lafaro KJ, et al. Institutional variation in recovery after cytoreductive surgery and hyperthermic intraperitoneal chemotherapy: an opportunity for enhanced recovery pathways. J Surg Oncol 2020; 122: 980-985.

\section{Address for correspondence}

Valeriia Volodymyrivna Chetverikova-Ovchynnyk

Odessa National Medical University

Odessa, Ukraine

e-mail: ovchinnik.val@yahoo.com

Submitted: 24.03 .2021

Accepted: 2.05.2021 